Document downloaded from:

http://hdl.handle.net/10251/69054

This paper must be cited as:

Escamilla Fuster, J.; Salido Gregorio, MA. (2016). A Dual Scheduling Model for Optimizing Robustness and Energy Consumption in Manufacturing Systems. Proceedings of the Institution of Mechanical Engineers, Part B: Journal of Engineering Manufacture. 1(1):1-12. doi:10.1177/0954405415625915

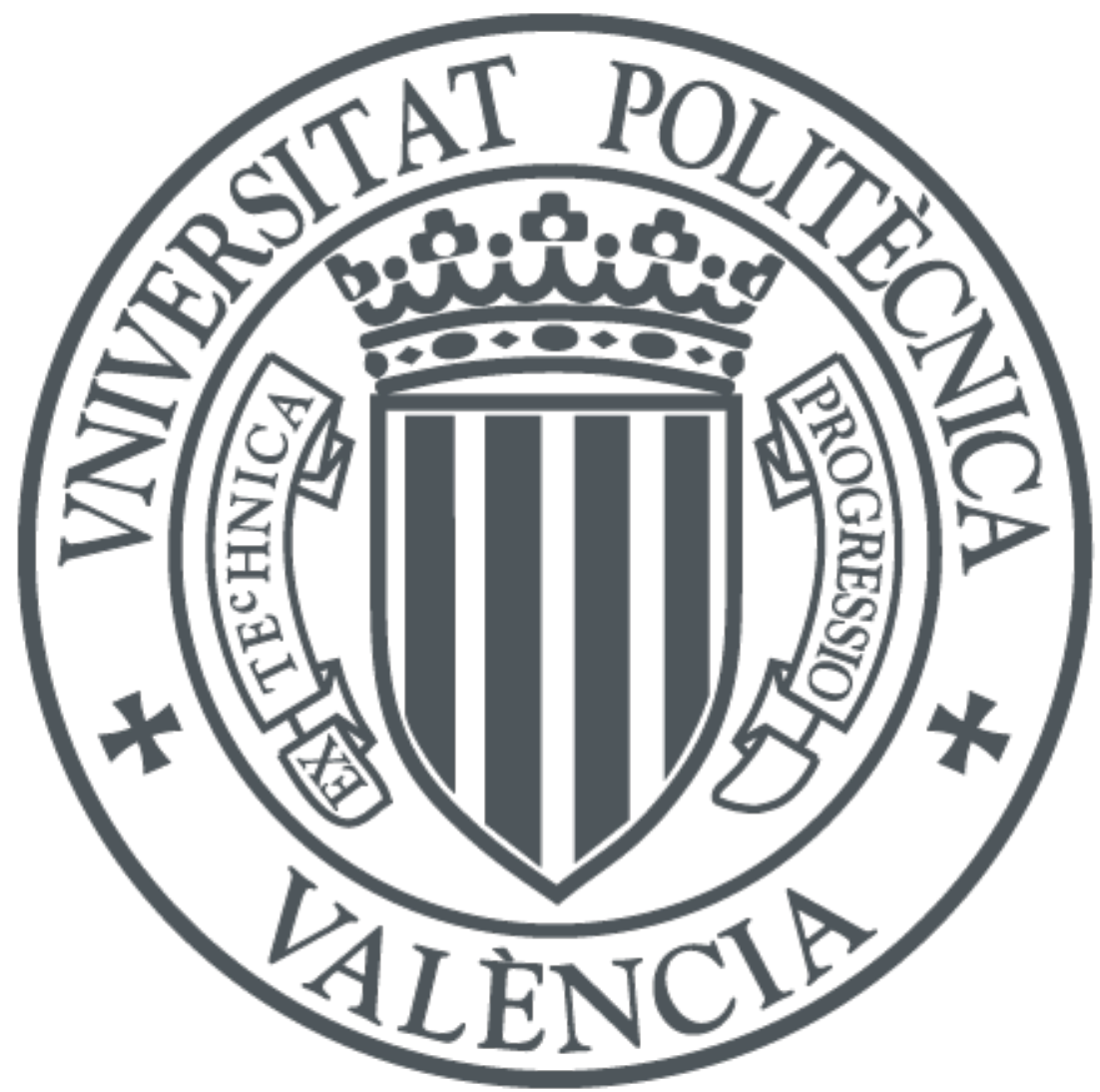

The final publication is available at

http://dx.doi.org/10.1177/0954405415625915

Copyright SAGE Publications (UK and US)

Additional Information 
See discussions, stats, and author profiles for this publication at: https://www.researchgate.net/publication/292673396

\section{A dual scheduling model for optimizing robustness and energy consumption in manufacturing systems}

Article in Proceedings of the Institution of Mechanical Engineers Part B Journal of Engineering Manufacture · February 2016 DOI: 10.1177/0954405415625915

CITATIONS

2 authors:

Joan Escamilla

Universitat Politècnica de València

13 PUBLICATIONS 72 CITATIONS

SEE PROFILE

\section{READS}

81

Miguel A. Salido

Universitat Politècnica de València

147 PUBLICATIONS 1,303 CITATIONS

SEE PROFILE

Some of the authors of this publication are also working on these related projects:

ROBUSTEZ, ESTABILIDAD DE MODELOS Y MÉTODOS DE OPTIMIZACIÓN DIFUSA APLICADOS A PROBLEMAS DE SCHEDULING View PROject

sustainability in future manufacturing systems View project 


\title{
A Dual Scheduling Model for Optimizing Robustness and Energy Consumption in Manufacturing Systems
}

():-

(CThe Author(s) 2010 Reprints and permission: sagepub.co.uk/journalsPermissions.nav DOI:10.1177/1081286510367554 http://mms.sagepub.com

\author{
Joan Escamilla and Miguel A. Salido \\ E-mail: jescamilla@dsic.upv.es,msalido@dsic.upv.es \\ Instituto de Automática e Informática Industrial \\ Universidad Politécnica de Valencia \\ Valencia, Spain
}

\begin{abstract}
Manufacturing systems involve a huge number of combinatorial problems that must be optimized in an efficient way. One of these problems is related to task scheduling problems. These problems are NP-hard, so most of the complete techniques are not able to obtain an optimal solution in an efficient way. Furthermore, most of real manufacturing problems are dynamic, so the main objective is not only to obtain an optimized solution in terms of makespan, tardiness, etc, but also to obtain a solution able to absorb minor incidences/disruptions presented in any daily process. Most of these industries are also focused on improving the energy-efficiency of their industrial processes. In this paper, we propose a knowledge-based model to analyze previous incidences occurred in the machines with the aim of modeling the problem to obtain robust and energy aware solutions. The resultant model (called dual model) will protect the more dynamic and disrupted tasks by assigning buffer times. These buffers will be used to absorb incidences during execution and to reduce the machine rate to minimize energy consumption. This model is solved by a memetic algorithm (GA*+LS) which combines a genetic algorithm (GA) with a local search (LS) to obtain robust and energy-aware solutions able to absorb further disruptions. The proposed dual model has been proven to be efficient in terms of energy consumption, robustness and stability in different and well-known benchmarks.
\end{abstract}

Keywords

Scheduling Problem, Energy Efficiency, Robustness, Dual Model.

\section{Introduction}

Nowadays, many companies and enterprises are focused on improving their benefits to be more competitive in their manufacturing processes. However, there exist other objectives beyond the benefits, such as robustness, sustainability, etc. Manufacturing processes have a huge impact on the environment. Some of them such as chemical, food, refining, paint or metal industries are highly energy consuming and presents a high risk of producing an important amount of waste or pollution. For example, during 2000, about $60 \%$ of the $\$ 700$ million energy expenditures in 37 U.S. automotive assembly 
plants are spent in painting processes [1]. In such a context, a 5\% reduction in energy consumption may result in a saving of more than half million dollars per year for each plant. Industrial processes are also known to be the major sources of greenhouse gas (GHG) emissions. Statistics has shown that the GHG emitted from the usage of energy sources such as electricity, coal, oil and gas during manufacturing accounts for more than $37 \%$ even $50 \%$ of the world total GHG [2].

Therefore, the enterprises have started to take steps to reduce GHG emission from their products and services under the mounting pressure stemming from the implementation of the Kyoto protocol and Copenhagen protocol. Industrial sector has a lot influence is the environmental impacts, because it represents the sector where more energy is used. The energy consumed in the world in 2011 was 524 quadrillion BTU and the energy used by the industrial amounts to 51\% [3]. So reducing consumption in this sector directly affects the total consumption. Nevertheless, it is still difficult for enterprises to consider renewable sources application and emissions reduction when making manufacturing and operation decisions, especially on the production planning and scheduling problems [4].

During the last few years, many works have been carried out to optimize energy consumption in manufacturing processes based on the machine level and the product level [5]. Besides, from the manufacturing system-level perspective, optimizing the objectives of production scheduling is a feasible and efficient approach for manufacturing companies to decrease energy consumption without any machine or product redesign. Recently, interesting efforts have been performed to investigate production-scheduling problems that take into account energy efficiency.

May et al. [6] addressed a multi-objective scheduling model related to energy consumption and makespan in a job-shop system and obtained a series of different Pareto front solutions based on a green genetic algorithm. Escamilla et al. [7] developed a genetic algorithm to minimize energy consumption and makespan in an extended version of the job-shop scheduling problem where each machine can work at different rates. Jiang et al. [8] built a multi-objective optimization model involving makespan, processing cost, processing quality and energy consumption for a flexible job-shop scheduling problem and designed a modified on-dominant sorting genetic algorithm to solve it. Liu et al. [9] established a scheduling model that minimized the total non-processing electricity consumption and total weighted tardiness for the job shop problem. This problem was solved by using a non-dominant sorting genetic algorithm.

In the literature on dynamic scheduling problems, predictive-reactive scheduling strategies have been widely used in manufacturing systems, where schedules are revised in response to dynamic factors [10]. Most of them only consider efficiency of the schedule like makespan. However, reducing energy consumption in production scheduling considering dynamic factors has been rather limited. Pach et al. [11] proposed a reactive scheduling model based on potential fields in flexible manufacturing systems, which considered three indicators: makespan, energy consumption and the number of resource switches in a dynamic context. Kum et. al [12] proposed a dynamic shop floor re-scheduling approach inspired by a neuroendocrine regulation mechanism.

The job-shop scheduling problem (JSP) represents a problem in which some tasks are assigned to machines with a specific processing time. We work with an extension of JSP where each machine can work at different rates, so each machine has different available processing times and energy consumptions. Thus, if a machine works at higher rate, it consumes more energy but the processing time is reduced. Energy-efficiency is a critical parameter to take into account, but also other parameters such as robustness can give to the schedule profitability, safety or simplicity to avoid rescheduling, so robustness and energy-efficiency can be considered in many real life manufacturing problems.

\section{Problem Description}

Many industrial processes can be represented as a job-shop scheduling problem where machines can work at different speeds/rates (JSMS). This problem consists of a set of $n$ jobs $\left\{J_{1}, \ldots, J_{n}\right\}$ and a set of $m$ machines $\left\{R_{1}, \ldots, R_{m}\right\}$. Each job $J_{i}$ consists of a sequence of $v_{i}$ tasks $\left(\theta_{i 1}, \ldots, \theta_{i v_{i}}\right)$. Each task $\theta_{i l}$ has a single machine requirement $R_{\theta_{i l}}$ and a start time 
$s t_{\theta_{i l}}$ to be determined. Each machine can work at different rates, so different processing times and energy consumptions can be applied to each task.

A feasible schedule is composed of a complete assignment of starting times to tasks that satisfies the following constraints:

1. The tasks of each job are sequentially scheduled.

2. Each machine can process at most one task at any time.

3. No preemption is allowed.

The aim of the JSMS problems is to find a feasible schedule that minimizes makespan and energy consumption meanwhile maximizes the robustness of the schedule.

This problem represents an extension of the standard job-shop scheduling problem $\left(J \| C_{\max }[13]\right)$. The association between duration and energy have been created so the JSMS problem can be denoted as $J($ Speed $) \| C_{\text {max }}$, Energy. For each task, three different speeds have been defined to the involve machine. Each speed of the machine has associated a duration and an energy consumption. When the working speed of a machine increases, the energy consumption also increases, but the duration of the involved task decreases.

\section{Robustness and stability}

Robustness can be related with many real world problems. In industry, robustness can be profitable, because if a perturbation appears in the schedule, it can remains valid so it's not necessary to reschedule and therefore to interrupt the production process. We consider that a system is robust, if it is able to maintain its functionality even though some incidences can appear. In scheduling problems a solution is robust if it's able to absorb some disruptions without the delay of further tasks.

In real world problem is very common to leave some gaps/buffers between consecutive tasks of the schedule. Sometimes new gaps appear to satisfy precedence constraints. In JSMS problem, there is the possibility to change the speed of the machines, so increasing the speed of a machine can be enough to recover the incidences (energy-efficiency buffer).

In JSMS problem, a solution $(S)$ is robust if given an incidence $(I)$, it only affects to one task, and it is able to be absorbed. In this case, the incidence can be absorbed by an existing gap or the machine that executes this task can increase the speed to absorb the incidence. Moreover a gap and an energy-efficiency buffer can be joined to absorb incidences. If there isn't any buffer or it is smaller than the incidence size, the delay of a task is propagated to other tasks, so the solution is not considered robust. Thus, the robustness of a solution can be modelled as:

- $\quad R_{S, I}=1$ iff only the affected task is modified by $I$. Thus a gap/buffer assigned to this task can absorb the incidence or the machine can increase its speed to absorb the incidence.

- $\quad R_{S, I}=0$ iff more tasks are affected by $I$. This means that the buffer assigned to this task cannot absorb the incidence and it is propagated to the rest of the schedule.

Thus, a robust solution is a solution that maintains its feasibility over the whole set of expected incidences. If a solution is not robust against a perturbation, it can be considered stable if it is able to remain valid against incidences with only few changes [14]. Thus, stability is an interest parameter for rescheduling in order to recover the original solution by means of modifying the value of only a few tasks.

Thus, we consider a solution $S$ is $s-$ stable if there is a solution $S^{\prime}$, such that $\left\|S^{\prime}-S\right\|<s$, where $\|$.$\| is some$ n-dimensional norm defined in the solution space to evaluate the difference between $S$ and $S^{\prime}$ [15]. So in our case, the difference between $S$ and $S^{\prime}$ can be the number of tasks that have to be changed to maintain feasibility. 


\section{Memetic Algorithm $\left(\mathrm{GA}^{*}+\mathrm{LS}\right)$}

In this section we present a memetic algorithm which combines a genetic algorithm (GA) with a local search (LS). Genetic Algorithms are adaptive methods which may be used to solve optimization problems [16]. They are based on the genetic process of biological organisms. Over many generations, natural populations evolve according to the principle of natural selection. At each generation, every new individual (chromosome) corresponds to a solution, that is, a schedule for the given JSMS instance. Before a GA can be run, a suitable encoding (representation) of the problem must be devised. The essence of a GA is to encode a set of parameters (known as genes) and to join them together to form a string of values (chromosome). A fitness function is also required, which assigns a figure of merit to each encoded solution. The fitness of an individual depends on its chromosome and is evaluated by the fitness function. During the run, parents must be selected for reproduction and recombined to generate offspring. Parents are randomly selected from the population, using a scheme which favours fitter individuals. Having selected two parents, their chromosomes are combined, typically by using crossover and mutation mechanisms to generate better offspring, so they represent better solutions. The process is iterated until a stopping criteria is satisfied.

\subsection{Chromosome encoding and decoding}

In genetic algorithms, each candidate represents a solution in the solution space. The first step to construct a GA is to define an appropriate genetic representation (coding). Table 1 shows an example of the processing time and the energy consumption of a JSMS composed of 3 jobs, 3 machines and 3 tasks per job. A chromosome is a permutation of the set

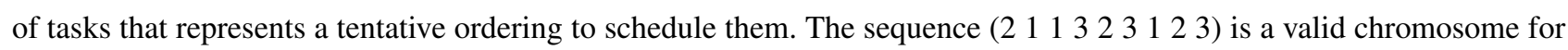
this example, where each number of the sequence represents the job of the task. The first number "2" represents the first task of the job 2 and it will be executed as soon as possible following the problem constraints. As it was shown in [17], this encoding has a number of interesting properties for the classic job-shop scheduling problem. However, in the JSMS problem, the machine speed of each operation has to be represented. Thus, it is added a value to each task in order to represent the speed of the machine that processes this task. For instance, a valid chromosome could be (2 $\underline{1} 1 \underline{2} 1 \underline{3} 3 \underline{3} 2 \underline{2} 3$ $\underline{2} 1 \underline{2} 2 \underline{3} 3 \underline{3}$ ) for the former example [7]. The speed of each task is showed with underline numbers. When the chromosome representation is decoded, each task starts as soon as possible following the precedence and machine constraints. With the machine speed representation, it can be calculated the processing time of each task and the energy consumption of the machine. The decoding result is shown in figure 5 .

\subsection{Initial population and Fitness}

Each gene represents one task of the problem. The position of each task determines its dispatch order in this genome/solution. The initial chromosomes are obtained following some dispatching rules or by random permutation. We employ six common dispatching rules: SPT (Shortest Processing Time), LPT (longest Processing Time), JML (Job with More Load), JMT (Job with More Tasks), MML (Machine with More Load) and MMT (Machine with More Tasks). We also employ a random rule, which select a job randomly from the remaining jobs. The dispatching rule SPT assigns high priority to short tasks, LPT assigns high priority to long tasks, JML assigns high priority to tasks which job has more load, JMT assigns high priority to tasks which job has more tasks, MML assigns high priority to tasks whose machine has more load and MMT assigns high priority to tasks whose machine has more tasks. To create each genome, each dispatching rule can be randomly selected with a probability of $10 \%$ and random rule with a probability of $40 \%$. Thus, it obtains variety and diversity in the initial population in order to obtain good solutions. Each dispatching rule assigns a priority to each task. This priority can be based on attributes of the jobs, the machines or tasks. The machine speed for each gene is generated depending on the value of $\lambda$. For $\lambda$ values lower than 0.6 the machine speed value is set to 1 , if $\lambda$ is higher than 0.6 the machine speed value is set to values 2 or 3 . 
In a single objective optimization, there is a single optimal solution. However, in multiobjective optimization there is not only an optimal solution and a set of optimal solutions can be identified. This set of optimal solutions composes the Pareto front. Diverse techniques have been developed to solve multiple objective optimization problems. One of the most well-known methods for solving multiple objective optimization problems is the Normalized Weighted Additive Utility Function (NWAUF), where multiple objectives are normalized and added to form a utility function. NWAUF has been implemented in wide range of MOO applications due to its simplicity and natural ability to identify efficient solutions. Let $f_{i}$ be the $i$ th objective function value. Then, the NWAUF is defined as:

$$
U_{j}=w_{1} f_{1}^{\prime}+w_{2} f_{2}^{\prime}+\ldots+w_{k} f_{k}^{\prime}
$$

where $w_{1}, w_{2}, \ldots, w_{k}$ are weights of importance and $f_{1}^{\prime}, f_{2}^{\prime}, \ldots, f_{k}^{\prime}$ are normalized values of $f_{1}, f_{2}, \ldots, f_{k}$. By normalizing different objectives, all objectives are evaluated in the same scale. Weights show decision maker's preference for each objective where, $\sum_{i=1}^{k} w_{i}=1$ and $0 \leq w_{i} \leq 1$ for $i=1, \ldots, k$. Using this utility function, the multiple objective optimizations can now be solved as a single objective optimization problem.

The definition of fitness function is just the reciprocal of the objective function value. The objective is to find a solution that minimizes the multi-objective makespan and energy consumption. Following NWAUF rules, the fitness function (2) is created, where the weights assigned to both variables are given by the $\lambda$ value. Since the values of energy consumption and makespan are not proportional, it is necessary to normalize both measures. Makespan is divided by MaxMakespan which is the maximum makespan value in a GA execution when $\lambda$ is equal to 0. MaxEnergy is the sum of the energy needed to execute all tasks at top speed.

$$
F=\left(\lambda * \frac{\text { Makespan }}{\text { MaxMakespan }}+(1-\lambda) * \frac{\text { SumEnergy }}{\text { MaxEnergy }}\right) * 100
$$

\subsection{Crossover operator}

For chromosome mating, the GA uses the Job-based Order Crossover (JOX) described in [18]. Given two parents, JOX selects a random subset of jobs and copies their genes to the offspring in the same positions as they are in the first parent. Then the remaining genes are taken from the second parent so as they maintain their relative ordering. To clarify how JOX works, let us consider the following two parents in Figure 1 for the example presented in section 6.

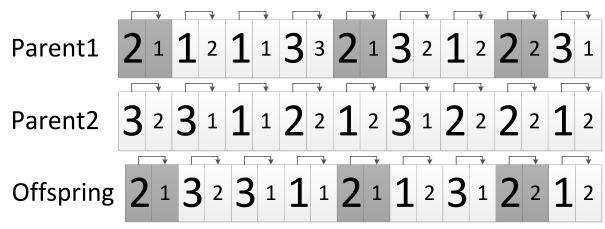

Fig. 1. Result of crossover operator between two parents

If the selected subset of jobs from the first parent just includes the job 2 (dark genes of parent 1 in Figure 1), the generated offspring is showed at the end of Figure 1.

Hence, operator JOX maintains for each machine a subsequence of operations in the same order as they are in Parent1 and the remaining in the same order as they are in Parent 2.

The remaining elements of GA are rather conventional. To create a new generation, all chromosomes from the current one are organized into couples which are mated two offsprings in accordance with the crossover probability. 


\subsection{Mutation operator}

Two offspring generated with crossover operation can also be mutated in accordance the mutation probability. Two position of chromosome child are randomly chosen (position "a" and position "b"), where "a" must be lower than "b". Values between "a" and "b" are randomly shuffled. Furthermore, in each gene, machine speed values are randomly changed. Finally, tournament replacement among every couple of parents and their offspring is carried out to obtain the next generation.

\subsection{Local Search}

Conventional GAs can produce good results. However, significant improvements can be obtained by hybridization with other methods. For example hybridization of a GA and a local search algorithm can produce better results because local search algorithms move from solution to solution in the search space by applying local changes. This is possible only if a neighbourhood relation is defined on the search space. Local search (LS) is implemented by defining a neighbourhood of each point in the search space as the set of chromosomes reachable by a given transformation rule. Then, a chromosome is replaced by the selected neighbour that satisfies the acceptance criterion. We propose a neighbourhood structure based on the concepts of critical path and critical block [19], [20] and [21]. A critical block is a maximal subsequence of operations of a critical path requiring the same machine. Mattfeld defined the neighbourhood structure $N_{1}$ for JSP [22]. It considers a set of moves called "interchange near the borderline of blocks on a single critical path", what means swapping pairs of operations only at the beginning or at the end of a critical block.

Following the idea of neighbourhood structure $N_{1}$ and the concepts of critical path and critical block, we have defined energy-efficiency neighbourhood structure $\left(N_{E E}\right)$ where each task in the critical path is analysed to check if the next tasks of the same machine are consecutive and involved in the critical path. If this condition is met, a new neighbour is created swapping both tasks and the machine speed is increased if its fitness is not worsened. Furthermore, when a task is not in a critical path, its speed is decreased to create a new neighbour. LS is only carried out if the runtime is bigger than the $80 \%$ of the given time-out.

\section{A Model for obtaining robust and energy-aware solutions}

In this section, we propose a knowledge-based model to obtain robust and energy-aware solutions for the problem job-shop scheduling problem with machines working at different speeds (JSMS).

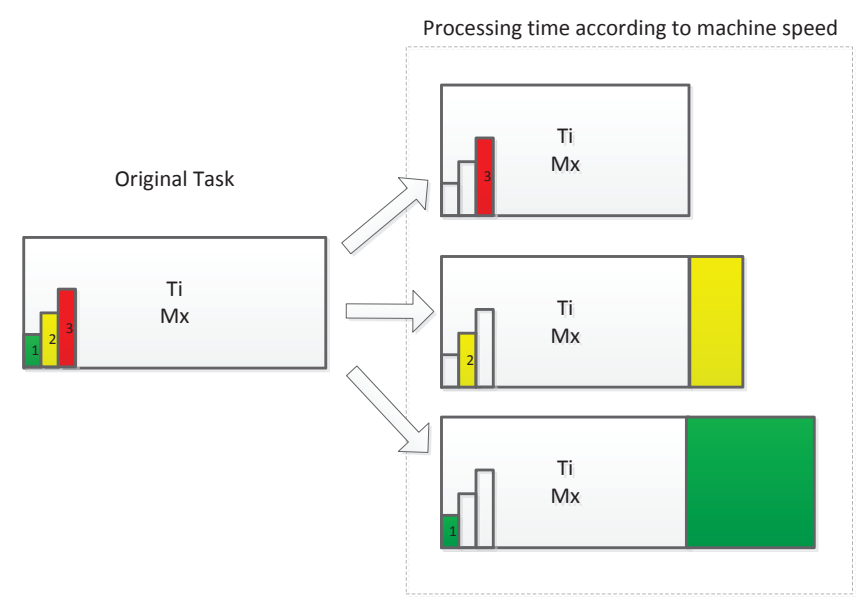

Fig. 2. Processing time of a task in a machine working at different rates 
Most scheduling problems involved in industrial processes are dynamic by nature. These dynamic problems are faced with incidences which generate delays in tasks. In some cases the original solution is recovered in a given time but in other cases the incidence is propagated in cascade so the original solution cannot be valid or recovered. Buffering is used in industrial processes to compensate for variations in the schedule. Changes in supply and demand would be an example of these variations. Think of buffering as a means to ensure that schedule continue running smoothly despite unforeseen factors, such as machine breakdowns, coming into play. Thus, buffers in industrial processes are usually inserted to keep operations running smoothly. However, the inclusion of buffer in scheduling processes has advantages and disadvantages. The most important advantage of using buffering in scheduling are, when done correctly, it's implementation tends to be more robust so thus to increase production efficiency, reduce overall costs and keep operations running smoothly. However, when done incorrectly, the opposite can be true. For example, if a schedule keeps too much buffer time between all tasks, the makespan increases up to remain the obtained solution not feasible in terms of profitability. To maintain an optimal balance, many operators tend to gravitate toward using industrial process strategies in which they use the least amount of buffering necessary to ensure their projected rates.

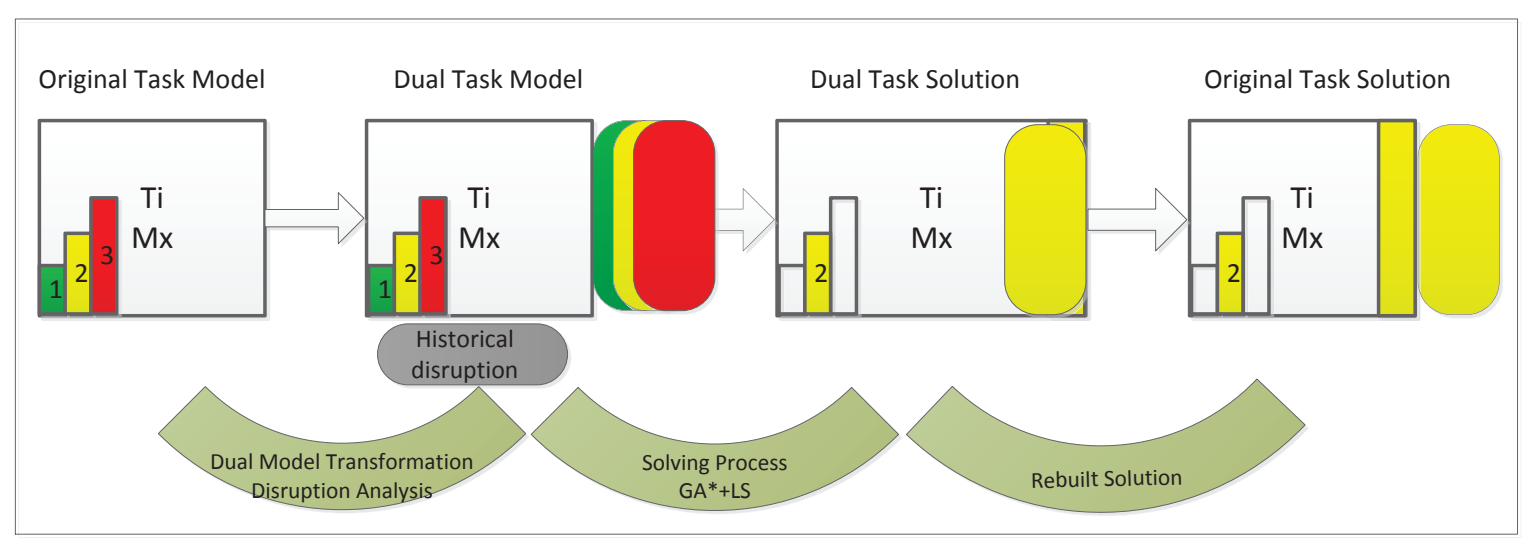

Fig. 3. Dual transformation of a scheduling task

In this section, we transform the original scheduling model into a dual scheduling model in which some selected tasks are accomplished of a buffer time according to previous problem knowledge. The size of each buffer time assigned to a task is in accordance to the average duration of the previous disruptions generated in this task.

Figure 2 shows the different rates a machine can work for an assigned task $T i$ : green colour (speed 1) means that the machine works at lowest rate (lowest energy consumption), so the processing time is highest; yellow colour (speed 2) means that the machine works at regular rate, so the processing time is regular; red colour (speed 3) means that the machine works at highest rate (highest energy consumption), so the processing time is lowest. Thus, the processing time of a task is dependent of the rate the assigned machine works. However, once the scheduling problem is solved and during execution, if an incidence occurs in task $T i$, the assigned machine could increase its rate (if it was planned to work at speed 1 or 2) in order to absorb this incidence. Thus, the time interval between a given processing time of task $T i$ and the lowest processing time of task $T i$ can be considered as an energy-efficient buffer.

The dual scheduling model is aimed to insert additional buffer times to more dynamic tasks. To this end, the historical data are analysed to determine which tasks must be protected by a buffer time. Due the above advantages and disadvantages of the inclusion of buffer times, only the most dynamic tasks will be selected. Figure 3 shows the dual transformation of a dynamic task $T i$. The dual transformation is composed of the following steps:

1. The original task model is composed of the number of tasks within the job (Ti), the assigned machine (Mx) and the different ways this machine can work (see figure 2). 
2. (Modeling) If the task $T i$ is selected among the most dynamic ones, a dual task model $\left(T i^{\prime}\right)$ is generated for this original task $T i$. Thus, three buffer times are generated to task $T i^{\prime}$ according to the three processing times that machine (Mx) can execute this dual task. Figure 4 shows possible combinations of three buffer times according to the size of the historical buffer time of original task $T i$. Algorithm 1 shows how to calculate the actual buffer time assigned to each dual task. As can be seen in Figure 4, the historical buffer time is completely assigned to the dual task if it is executed at highest speed (diff3). Depending of the processing time of the dual task for regular and lowest speed, the buffer time is calculated according to Algorithm 1. It can be observed in Figure 4, the three different cases: (a) the processing time at highest speed plus the historical buffer time is higher than the processing time at regular and lowest speed. In this case, all processing times are complemented with a buffer time in such a way that the sum of processing times and the assigned buffer time is equal in the three different speeds of the machine. The solver will be committed to select the green one (lowest speed) in order to minimize energy consumption. In Figure 4 (b), it can be observed that the processing time at highest speed plus the historical buffer time is higher than the processing time at regular but not at lowest speed. In this case only a buffer time is assigned to dual tasks with highest and regular speeds. In case (c) the processing time at highest speed plus the historical buffer time is lower than the processing time at regular and lowest speed. In this case only a buffer time is assigned to dual tasks with highest speed.

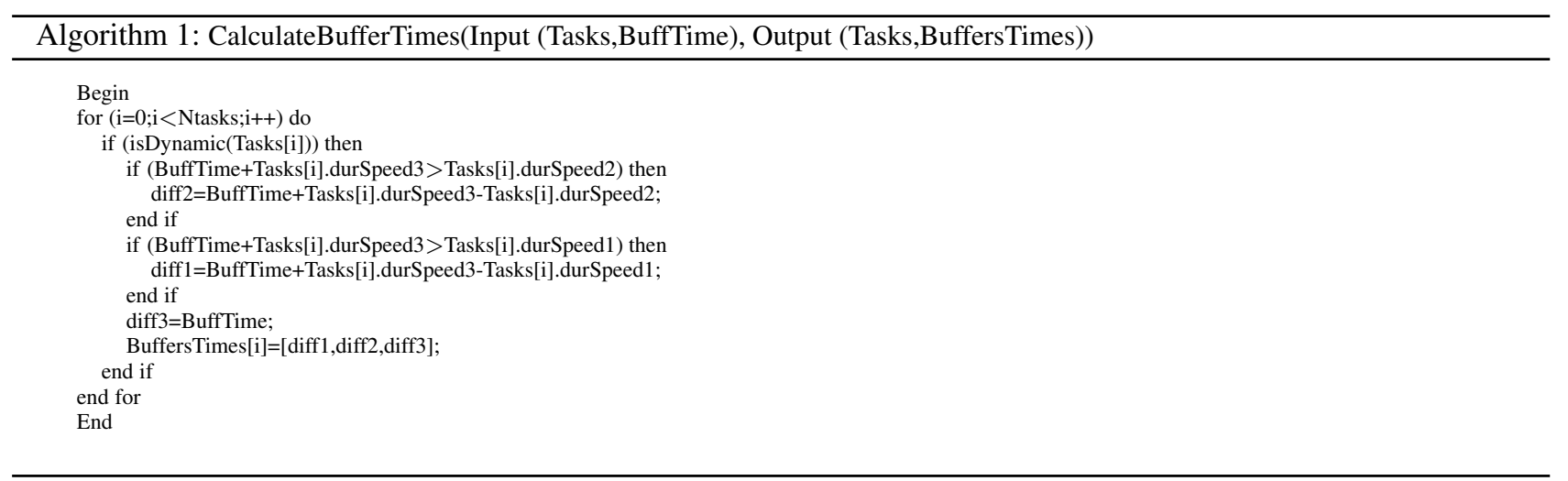

3. (Solving) Once the dual tasks $\left(T i^{\prime}\right)$ are generated, they are grouped with the rest of the original tasks $(T j)$ to generate the dual scheduling model. Our GA*+LS will be applied to obtain a Pareto front of the dual scheduling according to the parameter to be optimized: makespan and energy consumption. To this end, a convex combination between both parameters by a $\lambda \in[0,1]$ value determined the Pareto front. Formula 2 shows the bi-criteria objective. Both parameters are normalized to balance the weight of both when $\lambda$ gets values around 0,5 . In both cases each parameter is divided by a maximum value.

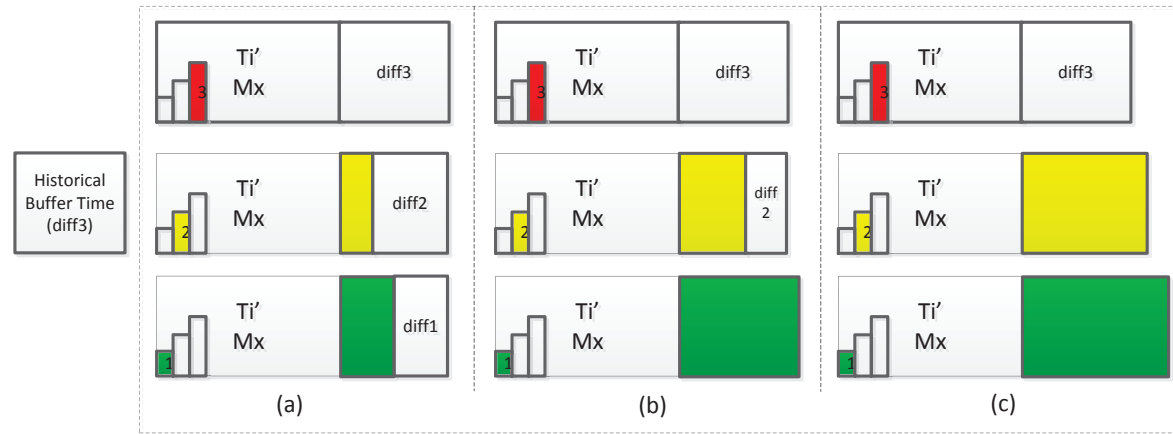

Fig. 4. Three different cases for the buffer time assignment. 
It must be taken into account that any solver could be applied to the dual model. The selected solver is aimed to assign for each task, its start time, the processing time and the speed that the machine executes each task. Furthermore the makespan and the energy consumption can be determined by the solver.

4. (Rebuilt) Once the obtained solutions are generated for the dual scheduling model, they are rebuilt to obtain solutions of the original scheduling model (see Figure 3). Thus, the robustness can be measured by decoupling the buffer times and the energy-efficiency buffers generated in the schedule. This rebuilt task is formally presented in Algorithm 2.

Sometimes buffers can be generated close to a buffer time assigned in the dual model. So if the sum of both is higher than the maximum duration of the historical incidence, the speed of the assigned machine can be reduced (if possible) to get a more energy-aware solution with the same degree of robustness.

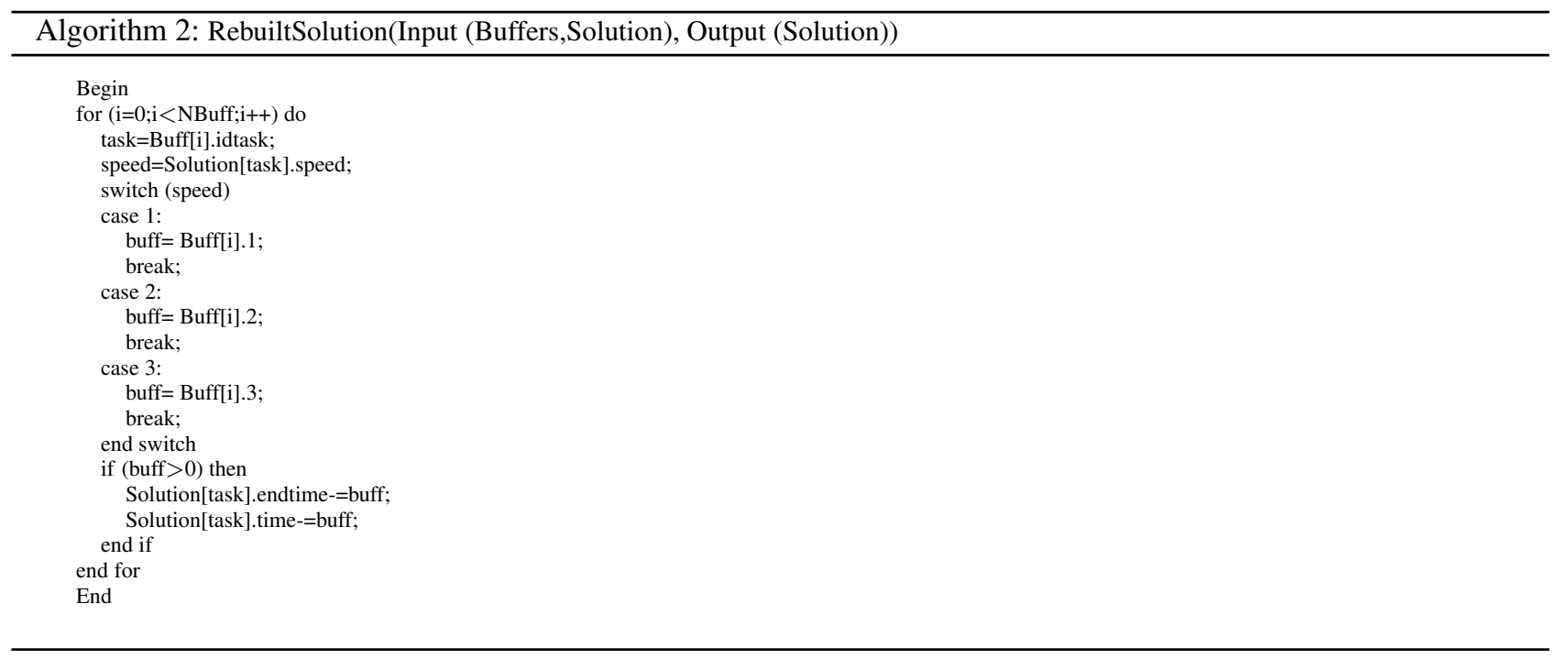

\section{Example}

In this section, a small example is shown to analyse and clarify the proposed model. Table 1 shows the processing time and the energy consumption for each rate/speed that each machine can work. The JSMS is composed of 3 jobs, 3 machines and 3 tasks per job. It must be taken into account that when the speed increases the energy consumption also increases but the processing time decreases. For this example, 60 incidences were randomly generated. Table 2 shows the distribution of incidences among the most dynamic tasks (Column NIncidences). Only the more dynamic ones are selected to generate a dual model (the 3 tasks with more incidences: Job1-Task1, Job1-Task2 and Job3-Task1 presented in Table 2).

The historical buffer time will be calculated according to the historical disruptions. In this above set of 60 incidences, the average size of the disruption was 2 . Thus, algorithm 1 is executed to each selected dynamic task. The input of the algorithm is composed of the selected task and the average size of disruptions. Finally, the dual task model is obtained. This dual task model is composed of the original tasks and their corresponding processing time, and the dual tasks with the original processing times plus the obtained buffer times shown in table 2.

For example, Job1-Task1' is a dual dynamic task with a buffer time assignment of type (b) according to Figure 4:

- Buffer time at lowest speed is 0: The processing time of the original task at highest speed plus the historical buffer time is not bigger than the processing time at lowest speed. 
Table 1. Example: Processing Time and Energy Used

\begin{tabular}{|l|c|c|c|c|c|c|}
\hline & \multicolumn{2}{|c|}{ Speed 1 } & \multicolumn{2}{c|}{ Speed 2 } & \multicolumn{2}{c|}{ Speed 3 } \\
\hline Tasks & Proc. Time & Energy & Proc. Time & Energy & Proc. Time & Energy \\
\hline Job1 Task1 & 11 & 5 & 9 & 6 & 8 & 7 \\
\hline Job1 Task2 & 10 & 4 & 9 & 6 & 7 & 7 \\
\hline Job1 Task3 & 9 & 4 & 8 & 5 & 5 & 6 \\
\hline Job2 Task1 & 8 & 3 & 7 & 4 & 6 & 5 \\
\hline Job2 Task2 & 7 & 3 & 6 & 4 & 4 & 5 \\
\hline Job2 Task3 & 8 & 4 & 7 & 5 & 5 & 7 \\
\hline Job3 Task1 & 6 & 2 & 5 & 3 & 4 & 4 \\
\hline Job3 Task2 & 7 & 3 & 6 & 4 & 4 & 5 \\
\hline Job3 Task3 & 6 & 2 & 5 & 3 & 3 & 4 \\
\hline
\end{tabular}

Table 2. Buffer Times for most dynamic tasks

\begin{tabular}{|c|c|c|c|c|}
\hline & & \multicolumn{3}{|c|}{ Buffer Times } \\
\hline Tasks & NIncidences & Speed 1 & Speed 2 & Speed 3 \\
\hline Job1-Task1' & 9 & 0 & 1 & 2 \\
\hline Job1-Task2' & 10 & 0 & 0 & 2 \\
\hline Job3-Task1' & 11 & 0 & 1 & 2 \\
\hline
\end{tabular}

- Buffer time at regular speed is 1: The processing time of the original task at highest speed plus the historical buffer time is 1 unit bigger than the processing time at regular speed.

- Buffer time at highest speed is 2: The processing time of the original task is increased with the historical buffer time (2 units).

Thus, the processing time of the dual tasks are updated by adding the calculated buffer times of Table 2. Then, the resultant dual model can be solved. Once the solution has been obtained, the dual tasks must rebuild to obtain the original processing times and their corresponding buffers.

Figure 5 shows the solution (Gantt chart) for the original example and the corresponding solution of the dual model. The assigned values of the processing time for the selected speed are shown in bold in table 1 . The buffer times are shown in grey blocks. The gaps are represented in black blocks. By comparing both solutions it can be observed that the energy consumption is the same and the makespan in the original model is 27 meanwhile in the dual model is 29 . However, the solution obtained by the dual model is more robust due to the fact that almost all tasks (except job2-Task3) are able to absorb incidences up to 2 time units.

\section{Evaluation}

In this section, an evaluation of the proposed dual model is carried out to analyse and compare against the original model for finding robust and energy-aware solutions. As it has been pointed out, a Memetic algorithm (GA*+LS) which combines a genetic algorithm (GA) with a local search (LS) to solve the JSMS is applied. Furthermore, a comparative study was carried out by changing the value of buffer times to demonstrate that, as the buffer time increases, the robustness and energy-efficiency also increase but the makespan worsens. Otherwise, stability is considered and solutions are analysed to know how incidences can be absorb with the robustness and stable concepts. 

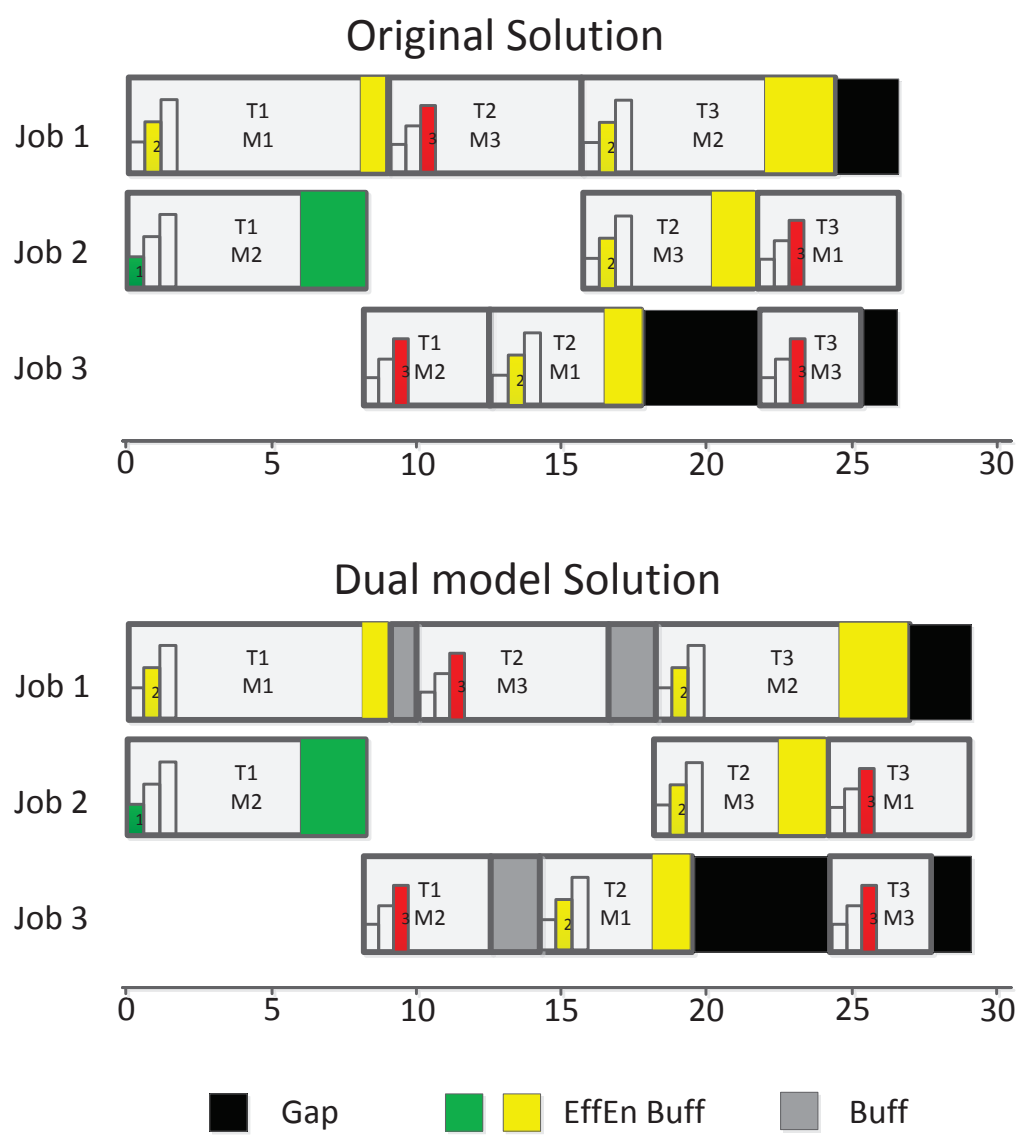

Fig. 5. Original solution and Dual model solution 
Table 3. Data information of Agnetis and Watson instances.

\begin{tabular}{|c|c|c|c|}
\hline Instances & Ntasks & Nincidences & NdynamicTasks \\
\hline 3_5_10 & 15 & 100 & 5 \\
\hline 5_10_50 & 30 & 200 & 10 \\
\hline 7_10_100 & 30 & 200 & 10 \\
\hline 3_20_50 & 60 & 400 & 20 \\
\hline 3_25_100 & 75 & 500 & 25 \\
\hline 3_30_200 & 90 & 600 & 30 \\
\hline Watson50 & 1000 & 7000 & 400 \\
\hline Watson100 & 2000 & 14000 & 800 \\
\hline Watson200 & 4000 & 28000 & 1600 \\
\hline
\end{tabular}

\subsection{Analysis of Parameters}

To carry out an extensive evaluation, two well-known benchmarks were used: Agnetis benchmarks [23] and Watson benchmarks [24]. In both cases, the instances were classified by the number of jobs $(j)$, the number of machines $(m)$, the number of tasks per job $\left(v_{\max }\right)$ and the maximum processing times $(p)$. In all Agnetis instances the number of jobs was 3 and the instances were represented as $m \_v_{\max \_} p$.

Agnetis instances are classified in two big groups small and large:

- Small: $j=3 ; m=3,5,7 ; v_{\max }=5,7,10 ; p=[1,10],[1,50],[1,100]$

- $\quad$ Large: $j=3 ; m=3 ; v_{\max }=20,25,30 ; p=[1,50],[1,100],[1,200]$

Watson instances the number of jobs $(j)$ changes in all case but $m, v_{\max }$ and $p$ remain equal:

- $\quad j=50,100,200 ; m=20 ; v_{\max }=20 ; p=[1,100]$

To simulate a historical background of the incidences for the Agnetis and Watson instances, a set of incidences were randomly assigned to tasks. To this end, the number of incidences was always proportional to the total number of tasks. Table 3 shows the analysed instances, the number of tasks, the number of simulated incidences and the number of selected dynamic tasks for the dual model. There must be a proportional relationship among the number of tasks, the number of simulated incidences and the number of selected dynamic tasks. The number of incidences must be significantly higher in order to classify and discriminate the more dynamic tasks. The number of dynamic tasks is also related to the number of tasks. A high number of dynamic tasks will generate a high degree of robustness but a worse makespan. As it was pointed out before, there must be equilibrium between the number of buffers and the optimality in terms of makespan.

For each type of problem, 10 instances were evaluated, so the average results are shown in next tables. Original instances were extended as explained in [7]. New instances and more information can be found in the research group webpage ${ }^{1}$.

\subsection{Comparative study}

In this section, it is compared the behaviour of the dual model against the original model. To this end, both models were executed over the same solver $\left(\mathrm{GA}^{*}+\mathrm{LS}\right)$ to analyse the performance of both models in terms of makespan, energy consumption and robustness. The incidences were randomly generated and assigned to tasks following the relation of table 3. Once an incidence is assigned to a task, the duration is randomly generated between 1 and $20 \%$ of the maximum processing time of this instance. Thus, for instances where processing time was 50, the incidences were assigned a duration between 1 and 10. Tables 4 show the results for the Agnetis instances 3_5_10 and 3_25_100 and for Watson100 instances.

\footnotetext{
${ }^{1}$ http://gps.webs.upv.es/jobshop/
} 
Table 4. Comparative study between Dual Model and Original Model with average duration of the incidences

\begin{tabular}{|c|c|c|c|c|c|c|c|c|c|}
\hline 3_5_10 & \multicolumn{3}{|c|}{ Dual Model (AvgInc)+ GA*+LS } & \multicolumn{3}{c|}{ Original Model + GA*+LS } & Comparative \\
\hline$\lambda$ & Mk & Energy & F & \%Rob & Mk & Energy & F & \%Rob & Diff \%Rob \\
\hline 0 & 66 & 84.4 & 55.37616 & 98.8 & 65.8 & 84.4 & 55.37616 & 97.6 & 1.2 \\
\hline 0.1 & 65.4 & 84.5 & 55.67521 & 98.8 & 65.2 & 84.5 & 55.65811 & 98.1 & 0.7 \\
\hline 0.2 & 64.6 & 84.7 & 55.90445 & 98.8 & 64.4 & 84.7 & 55.87027 & 98.2 & 0.6 \\
\hline 0.3 & 63.3 & 85.3 & 56.02253 & 98 & 63.2 & 85.2 & 55.94217 & 97.1 & 0.9 \\
\hline 0.4 & 60.2 & 87.8 & 55.86224 & 94 & 59.7 & 88.1 & 55.78161 & 92.5 & 1.5 \\
\hline 0.5 & 55.8 & 92.5 & 54.97107 & 88 & 54 & 94.2 & 54.72392 & 83.8 & 4.2 \\
\hline 0.6 & 52.4 & 98.2 & 53.50526 & 81.1 & 48.6 & 104 & 52.98928 & 71.1 & 10 \\
\hline 0.7 & 49.2 & 106 & 51.25795 & 75.3 & 45.2 & 112.1 & 50.02799 & 61.2 & 14.1 \\
\hline 0.8 & 46.1 & 117.8 & 48.10526 & 68.7 & 42.2 & 123.6 & 46.16438 & 51.2 & 17.5 \\
\hline 0.9 & 45.6 & 122.7 & 44.35071 & 68.5 & 41 & 133.6 & 41.46373 & 38.6 & 29.9 \\
\hline 1 & 45.5 & 152 & 40.23035 & 65.9 & 41 & 152 & 36.30501 & 29.6 & 36.3 \\
\hline
\end{tabular}

\begin{tabular}{|c|c|c|c|c|c|c|c|c|c|}
\hline 3_25_100 & \multicolumn{2}{|c|}{ Dual Model (AvgInc)+ GA*+LS } & \multicolumn{3}{|c|}{ Original Model + GA*+LS } & Comparative \\
\hline$\lambda$ & Mk & Energy & F & \%Rob & Mk & Energy & F & \%ob & Diff \%Rob \\
\hline 0 & 2908.7 & 3827.1 & 53.35316 & 99.80 & 2894.2 & 3827.1 & 53.35316 & 91.80 & 8.00 \\
\hline 0.1 & 2791.6 & 3827.7 & 53.79550 & 98.30 & 2782.3 & 3827.6 & 53.77443 & 91.58 & 6.72 \\
\hline 0.2 & 2750.4 & 3840.5 & 54.19934 & 97.26 & 2753 & 3839 & 54.19750 & 91.76 & 5.50 \\
\hline 0.3 & 2642.1 & 3899.8 & 54.44719 & 95.32 & 2632.7 & 3903.4 & 54.41884 & 90.14 & 5.18 \\
\hline 0.4 & 2529.1 & 3993 & 54.33040 & 93.26 & 2516.7 & 4005.1 & 54.32539 & 89.74 & 3.52 \\
\hline 0.5 & 2380.1 & 4182.5 & 53.76294 & 86.22 & 2334.2 & 4216.7 & 53.53502 & 83.00 & 3.22 \\
\hline 0.6 & 2147.6 & 4573.4 & 52.17626 & 76.04 & 2129.6 & 4543.6 & 51.77883 & 75.20 & 0.84 \\
\hline 0.7 & 2022 & 4906.1 & 49.80327 & 69.20 & 1919.4 & 5123.7 & 49.25210 & 61.44 & 7.76 \\
\hline 0.8 & 1899.9 & 5451.8 & 46.67197 & 59.72 & 1806.2 & 5671.6 & 45.72355 & 50.04 & 9.68 \\
\hline 0.9 & 1839.2 & 5943.9 & 42.53255 & 53.64 & 1725.9 & 6261.1 & 40.84004 & 36.72 & 16.92 \\
\hline 1 & 1816.6 & 6948.4 & 37.57725 & 48.18 & 1692.5 & 7084.6 & 34.99826 & 25.74 & 22.44 \\
\hline
\end{tabular}

\begin{tabular}{|c|c|c|c|c|c|c|c|c|c|}
\hline Watson100 & \multicolumn{3}{|c|}{ Dual Model (AvgInc)+ GA*+LS } & \multicolumn{3}{c|}{ Original Model + GA*+LS } & Comparative \\
\hline$\lambda$ & Mk & Energy & $\mathrm{F}$ & \%Rob & Mk & Energy & $\mathrm{F}$ & \%Rob & Diff \%Rob \\
\hline 0 & 12324.9 & 105478.1 & 53.06175 & 100.00 & 12354.7 & 105478.1 & 53.06175 & 93.74 & 6.26 \\
\hline 0.1 & 12080.4 & 105480.1 & 55.88896 & 100.00 & 12047 & 105478.1 & 55.86523 & 93.46 & 6.54 \\
\hline 0.2 & 12037.6 & 105509.6 & 58.66911 & 99.98 & 12024.9 & 105502.8 & 58.64981 & 93.43 & 6.55 \\
\hline 0.3 & 12015.3 & 105699.9 & 61.48584 & 99.85 & 11979.6 & 105806.4 & 61.45107 & 93.37 & 6.47 \\
\hline 0.4 & 11868.3 & 106408.8 & 64.07627 & 99.49 & 11813.5 & 106537.2 & 63.96736 & 93.11 & 6.38 \\
\hline 0.5 & 11619.4 & 108681.5 & 66.44313 & 97.97 & 11557 & 109370.6 & 66.4093 & 91.63 & 6.34 \\
\hline 0.6 & 9013.5 & 141328.7 & 64.84557 & 75.91 & 8692.1 & 146631.3 & 64.61636 & 72.36 & 3.55 \\
\hline 0.7 & 8873.8 & 144645 & 63.64502 & 73.70 & 8537.6 & 150891.8 & 63.00417 & 69.19 & 4.51 \\
\hline 0.8 & 8739 & 147060.5 & 61.85831 & 71.63 & 8421.7 & 153772 & 60.82655 & 66.21 & 5.42 \\
\hline 0.9 & 8706.2 & 148108.1 & 60.20297 & 70.71 & 8419.4 & 153626.4 & 58.73666 & 67.41 & 3.30 \\
\hline 1 & 7932.1 & 195038.4 & 53.39947 & 45.23 & 7306.7 & 190808.3 & 49.18773 & 23.68 & 21.55 \\
\hline
\end{tabular}


Table 5. Comparative between dual model with MaxInc and dual model with AvgInc

\begin{tabular}{|c|c|c|c|c|c|c|c|c|c|}
\hline 7_10_100 & \multicolumn{2}{|c|}{ Dual Model (MaxInc) + GA*+LS } & \multicolumn{3}{|c|}{ Dual Model (AvgInc)+ GA*+LS } & Comparative \\
\hline$\lambda$ & Mk & Energy & F & \%Rob & Mk & Energy & F & \%Rob & Diff \%Rob \\
\hline 0 & 1008.9 & 1571.4 & 53.36161 & 97.80 & 1006.6 & 1571.4 & 53.36161 & 97.30 & 0.50 \\
\hline 0.1 & 1002.9 & 1572.5 & 54.11091 & 97.65 & 1000.6 & 1572.5 & 54.09746 & 97.15 & 0.50 \\
\hline 0.2 & 991 & 1576.4 & 54.79191 & 96.85 & 988.5 & 1576.4 & 54.76258 & 96.60 & 0.25 \\
\hline 0.3 & 933 & 1610.1 & 55.20634 & 94.40 & 928.9 & 1611.2 & 55.14574 & 93.25 & 1.15 \\
\hline 0.4 & 901.5 & 1641 & 55.26405 & 92.10 & 894.4 & 1642.3 & 55.12247 & 89.55 & 2.55 \\
\hline 0.5 & 868.4 & 1687.1 & 54.92773 & 88.05 & 853.7 & 1703.4 & 54.75819 & 85.00 & 3.05 \\
\hline 0.6 & 812.6 & 1817.3 & 54.16006 & 81.55 & 801 & 1823.1 & 53.85088 & 77.70 & 3.85 \\
\hline 0.7 & 764.5 & 1983.5 & 52.59537 & 71.35 & 744.4 & 2006 & 51.98095 & 66.95 & 4.40 \\
\hline 0.8 & 720.8 & 2203.5 & 49.88613 & 63.00 & 683.1 & 2314.9 & 48.83531 & 53.15 & 9.85 \\
\hline 0.9 & 709.6 & 2323.9 & 46.56537 & 62.00 & 673.1 & 2420.3 & 44.89425 & 49.25 & 12.75 \\
\hline 1 & 705.9 & 2935.1 & 42.72285 & 62.20 & 671.3 & 2935.1 & 40.62202 & 40.50 & 21.70 \\
\hline
\end{tabular}

\begin{tabular}{|c|c|c|c|c|c|c|c|c|c|}
\hline Watson50 & \multicolumn{2}{|c|}{ Dual Model (MaxInc) + GA + LS } & \multicolumn{3}{c|}{ Dual Model (AvgInc)+ GA + LS } & Comparative \\
\hline$\lambda$ & Mk & Energy & $\mathrm{F}$ & $\%$ Rob & Mk & Energy & F & \%ob & Diff \%Rob \\
\hline 0 & 7252.9 & 53631.5 & 53.40844 & 100.00 & 7253.7 & 53631.5 & 53.40844 & 100.00 & 0.00 \\
\hline 0.1 & 7035.8 & 53631.5 & 55.53904 & 100.00 & 7019.8 & 53637.3 & 55.52724 & 99.99 & 0.01 \\
\hline 0.2 & 6994.7 & 53694.2 & 57.63237 & 99.97 & 6985.5 & 53675.8 & 57.59795 & 99.91 & 0.05 \\
\hline 0.3 & 6960 & 53754.4 & 59.64355 & 99.86 & 6910.7 & 53877.4 & 59.57130 & 99.71 & 0.15 \\
\hline 0.4 & 6886.9 & 54071.2 & 61.55995 & 99.67 & 6821 & 54422.1 & 61.49003 & 98.95 & 0.72 \\
\hline 0.5 & 6749 & 54848.6 & 63.14064 & 98.76 & 6690.5 & 55244.8 & 63.02893 & 97.97 & 0.79 \\
\hline 0.6 & 5711.4 & 65252.4 & 62.38081 & 88.64 & 5411 & 68906.6 & 61.92607 & 80.55 & 8.08 \\
\hline 0.7 & 5459.9 & 68755.2 & 61.12227 & 85.20 & 5185.6 & 72321.3 & 60.14895 & 75.37 & 9.83 \\
\hline 0.8 & 5363.4 & 70593.5 & 59.62423 & 83.45 & 5078.9 & 74153.8 & 57.91645 & 72.34 & 11.11 \\
\hline 0.9 & 5302.5 & 71753.7 & 57.82274 & 82.05 & 5030.8 & 75624.5 & 55.61081 & 70.41 & 11.64 \\
\hline 1 & 4945.9 & 95791.2 & 52.52671 & 70.55 & 4633.3 & 98728.8 & 49.20303 & 47.29 & 23.26 \\
\hline
\end{tabular}

In all cases, the experiments were made over 10 different instances, so the values of the tables show average results. The values of robustness were also an average over the all incidences, so they are shown as a percentage.

Table 4 shows the makespan, energy consumption, F value and robustness of both models for different lambda values (see equation 2). Thus, the Pareto front can be obtained for the multiobjective problem (makespan vs. energy consumption). It must be taken into account that $\lambda=0$ means that only energy consumption is taken into account so makespan is not considered, meanwhile $\lambda=1$ means that only makespan is considered. In all analysed cases, it can be observed that makespan was always lower for the original model than the dual model. This is due to the fact that buffer times were inserted in the dual model to increase robustness, so the makespan was increased. For instance, the results for 3_5_10 in table 4 , for $\lambda=1$ (only makespan is taken into account) the average makespan for the original model was 41 meanwhile the average makespan for the dual model was 45.5. In contrast the value of robustness for the original model was $29.6 \%$ and the robustness for the dual model was $65.9 \%$. So, with this instance, it is shown that losing 4.5 units of makespan, the robustness was increased $36.3 \%$. Something similar happens when only energy consumption was taken into account. However, the energy consumption was lower for the dual model than for the original model in most instances, mainly for $\lambda>0.5$. This is because the generated buffers during the solving process plus the previously assigned buffer times allowed machines to decrease their speed in the rebuilt phase.

In general, the solutions obtained by applying the dual model had a worse F value, but on other hand the solutions were more robust. Furthermore, it can be observed in the last column (Diff \%Rob), that as the lambda increased the difference in robustness increased in a significant way. So, with the application of our dual model, it can be concluded that:

- When only makespan was optimized, makespan was worsened but the robustness was improved.

- When only the energy consumption was optimized, the robustness was improved without lose optimality. 
Table 6. Study of stability

\begin{tabular}{|c|c|c|c|c|}
\hline 3_25_100 & \multicolumn{4}{|c|}{ Dual Model (AvgInc)+ GA*+LS } \\
\hline$\lambda$ & \% Rob & \% S1 & \% S2 & \% Total Stability \\
\hline 0 & 99.80 & 0.20 & 0.00 & 100.00 \\
\hline 0.1 & 98.30 & 0.80 & 0.74 & 99.84 \\
\hline 0.2 & 97.26 & 1.38 & 0.88 & 99.52 \\
\hline 0.3 & 95.32 & 1.48 & 2.06 & 98.86 \\
\hline 0.4 & 93.26 & 1.68 & 3.06 & 98.00 \\
\hline 0.5 & 86.22 & 3.34 & 4.70 & 94.26 \\
\hline 0.6 & 76.04 & 4.70 & 6.74 & 87.48 \\
\hline 0.7 & 69.20 & 5.46 & 6.42 & 81.08 \\
\hline 0.8 & 59.72 & 5.22 & 5.40 & 70.34 \\
\hline 0.9 & 53.64 & 5.60 & 4.40 & 63.64 \\
\hline 1 & 48.18 & 9.24 & 6.64 & 64.06 \\
\hline
\end{tabular}

\begin{tabular}{|c|c|c|c|c|}
\hline Watson50 & \multicolumn{4}{|c|}{ Dual Model (AvgInc)+ GA*+LS } \\
\hline$\lambda$ & \% Rob & \% S1 & \% S2 & \% Total Stability \\
\hline 0 & 100.00 & 0.00 & 0.00 & 100.00 \\
\hline 0.1 & 99.99 & 0.01 & 0.00 & 100.00 \\
\hline 0.2 & 99.91 & 0.07 & 0.00 & 99.98 \\
\hline 0.3 & 99.71 & 0.17 & 0.05 & 99.93 \\
\hline 0.4 & 98.95 & 0.52 & 0.15 & 99.62 \\
\hline 0.5 & 97.97 & 1.09 & 0.23 & 99.29 \\
\hline 0.6 & 80.55 & 8.31 & 2.34 & 91.20 \\
\hline 0.7 & 75.37 & 9.93 & 2.55 & 87.85 \\
\hline 0.8 & 72.34 & 10.57 & 2.66 & 85.57 \\
\hline 0.9 & 70.41 & 10.72 & 2.66 & 83.78 \\
\hline 1 & 47.29 & 17.02 & 4.42 & 68.73 \\
\hline
\end{tabular}

- When both variables were optimized, makespan was worse but energy consumption had a different behaviour, but robustness was improved.

Table 5 shows the results of Agnetis instances 7_10_100 and Watson50 instances to analyse the behaviour of the dual model over the size of the buffer times. To this end, two different buffer time sizes were analysed: buffer times with the maximum duration size of the incidences (MaxInc) and buffer times with the average size of all incidences (AvgInc). This table represents the behaviour of all instances analysed in Table 3 and all instances showed a similar behaviour.

The dual model with MaxInc had an F value higher than the dual model with AvgInc. However, the robustness value was always higher in the dual model with MaxInc. This is because, as the buffer time of the MaxInc was always bigger than AvgInc, the makespan value was also higher and thus the F value. For example for $\lambda=1$ in table 5-7_10_100, it can be seen that the difference between both makespan was 34.6 but the difference between robustness was $21.70 \%$.

Finally, the stability was analysed for the dual model in both Agnetis and Watson instances. As it was pointed out in section 3, stability is the ability of a solution to recover with only a few changes. Thus, given an incidence in a task $T i$, 0 -stable is equivalent to robustness, so no further tasks will be affected, meanwhile 1-stable and 2-stable require to change the start time of $1(T j)$ and 2 tasks $(T k, T p)$, respectively.

Table 6 shows the percentage of robustness or 0-stability (\%Rob), 1-stability (\%S1) and 2-stability (\%S2), and the percentage of total stability (\%Total Stability) obtained by summing up the three previous parameters for Agnetis instances 3_25_100 and Watson50 instances. The rest of instances presented in Table 3 maintained similar behaviours. It can be observed that robustness or 0 -stability (\%Rob) had a higher value than 1-stability (\%S1) and 2-stability (\%S2). This is due to the fact that, the dual model was designed to add buffer times to more dynamic tasks. Thus, given an incidence, the algorithm tried to absorb it by increasing the speed of the machine or by using the assigned buffer time. If the incidence was 
not able to be absorbed, it was checked whether the incidence could be absorbed by the next task of the same machine or by the next task of the same job. If the incidence can be absorbed by changing only one task (from the same machine or the same job), it is considered a 1-stable solution. However, if both tasks have to be modified, it is considered a 2-stable solution. For both Agnetis and Watson instances, the percentage of total stability was close to $100 \%$ for lambda values lower than 0.5 . For example, when $\lambda=0$, all incidences were absorbed with 0,1 or 2 changes in the original solutions, achieving a $100 \%$ of total stability in both instances. Furthermore, as lambda values increased (the makespan was minimized), the percentage of robustness decreased and more incidences were not able to be absorbed. However, the 1-stability and 2-stability increased to absorb some of the incidences that were not directly absorbed by the dual model. For instance, in Watson50 instances, for $\lambda=1$ percentage of total stability increased a $21.44 \%$ with respect to robustness (47.29\%), achieving a total percentage of stability of $68.73 \%$.

\section{Conclusion}

Industrial processes involve a large number of task scheduling problems. Job-shop scheduling problems where machines can work at different rates/speeds represent a large number of combinatorial problems in industrial processes. In this paper, a dual model is proposed to relate optimality criteria with energy consumption and robustness/stability. This model is committed to protect dynamic tasks against further incidences in order to obtain robust and energy-aware solutions. The proposed dual model has been evaluated with a memetic algorithm $\left(\mathrm{GA}^{*}+\mathrm{LS}\right)$ to compare the behaviour against the original model. The result shows that the proposed dual model was able to obtain more robust solutions in all instances and more energy efficient solutions in most of them. This is due to the fact that the assigned buffer times to some tasks are used to protect them against incidences. Furthermore, if during execution, there is no incidence, the involved machine can use this buffer to work at lower speed, so the energy consumption can be also reduced. The combination of robustness and stability gives the proposal an added value because although an incidence cannot be directly absorbed by the disrupted task, it can be repaired by involving only a small number of tasks. Thus the original solution can be recovered in order to maintain feasible the rest of the obtained schedule.

\section{Acknowledgment}

This research has been supported by the Spanish Government under research project TIN2013-46511-C2-1 for the Spanish government and the TETRACOM EU project FP7-ICT-2013-10-Nº 609491.

\section{References}

[1] Guorong Chen, Liang Zhang, Jorge Arinez, and Stephan Biller. Energy-efficient production systems through schedule-based operations. Automation Science and Engineering, IEEE Transactions on, 10(1):27-37, 2013.

[2] Stephen T Newman, A Nassehi, R Imani-Asrai, and V Dhokia. Energy efficient process planning for cnc machining. CIRP Journal of Manufacturing Science and Technology, 5(2):127-136, 2012.

[3] U.S. Energy Information Administration. International energy outlook. Journal of Scheduling, 2013.

[4] Xiaoqing Wang, Hongwei Ding, Minmin Qiu, and Jin Dong. A low-carbon production scheduling system considering renewable energy. In Service Operations, Logistics, and Informatics (SOLI), 2011 IEEE International Conference on, pages 101-106. IEEE, 2011.

[5] Yan He, Yufeng Li, Tao Wu, and John W Sutherland. An energy-responsive optimization method for machine tool selection and operation sequence in flexible machining job shops. Journal of Cleaner Production, 87:245-254, 2015.

[6] Gökan May, Bojan Stahl, Marco Taisch, and Vittal Prabhu. Multi-objective genetic algorithm for energy-efficient job shop scheduling. International Journal of Production Research, (ahead-of-print):1-19, 2015. 
[7] Joan Escamilla, Miguel A Salido, Adriana Giret, and Federico Barber. A metaheuristic technique for energy-efficiency in job-shop scheduling. Workshop on Constraint Satisfaction Techniques (COPLAS) 2014: 24th International Conference on Automated Planning and Scheduling (ICAPS), pages 42-50, 2014.

[8] E. Mingcheng Z. Jiang, L. Zuo. Study on multi-objective flexible job-shop scheduling problem considering energy consumption. Journal of Industrial Engineering and Management, 3(7):589-604, 2014.

[9] Ying Liu, Haibo Dong, Niels Lohse, Sanja Petrovic, and Nabil Gindy. An investigation into minimising total energy consumption and total weighted tardiness in job shops. Journal of Cleaner Production, 65:87-96, 2014.

[10] S. Petrovic D. Ouelhadj. A survey of dynamic scheduling in manufacturing systems. Journal of Industrial Engineering and Management, 4(12):417-431, 2009.

[11] Cyrille Pach, Thierry Berger, Yves Sallez, Thérèse Bonte, Emmanuel Adam, and Damien Trentesaux. Reactive and energy-aware scheduling of flexible manufacturing systems using potential fields. Computers in Industry, 65(3):434-448, 2014.

[12] Kun Zheng, Dunbing Tang, Adriana Giret, Wenbin Gu, and Xing Wu. Dynamic shop floor re-scheduling approach inspired by a neuroendocrine regulation mechanism. Proceedings of the Institution of Mechanical Engineers, Part B: Journal of Engineering Manufacture, page 0954405414558699, 2015.

[13] J. Blazewicz, W. Cellary, R. Slowinski, and J. Weglarz. Scheduling under resource constraints-deterministic models. Annals of Operations Research, 7:1-356, 1986.

[14] E. Jen. Stable or robust? whats the difference? Complexity, 8(3):12-18, 2003.

[15] Federico Barber and Miguel A Salido. Robustness, stability, recoverability, and reliability in constraint satisfaction problems. Knowledge and Information Systems, 44(3):719-734, 2015.

[16] D. Beasley, RR Martin, and DR Bull. An overview of genetic algorithms: Part 1. fundamentals. University computing, 15:58-58, 1993.

[17] Ramiro Varela, David Serrano, and María Sierra. New codification schemas for scheduling with genetic algorithms. In Artificial Intelligence and Knowledge Engineering Applications: A Bioinspired Approach, pages 11-20. Springer, 2005.

[18] C Bierwirth. A generalized permutation approach to jobshop scheduling with genetic algorithms. OR Spectrum, 17:87-92, 1995.

[19] Hirofumi Matsuo, Chank Juck Suh, and Robert S Sullivan. A controlled search simulated annealing method for the general jobshop scheduling problem. Austin, TX: Grad. School of Bus., Univ. of Texas, 1988.

[20] Peter JM Van Laarhoven, Emile HL Aarts, and Jan Karel Lenstra. Job shop scheduling by simulated annealing. Operations research, 40(1):113-125, 1992.

[21] E Nowicki and C Smutnicki. A fast taboo search algorithm for the job shop scheduling problem. Management Science, 42:797-813, 1996.

[22] D. C Mattfeld. Evolutionary search and the job shop investigations on genetic algorithms for production scheduling. Springer-Verlag, 1995.

[23] A. Agnetis, M. Flamini, G. Nicosia, and A. Pacifici. A job-shop problem with one additional resource type. Journal of Scheduling, 14(3):225-237, 2011.

[24] Jean-Paul Watson, Laura Barbulescu, Adele E Howe, and L Darrell Whitley. Algorithm performance and problem structure for flow-shop scheduling. In AAAI/IAAI, pages 688-695, 1999. 